\title{
Évolutions du niveau d'eau en bassin semi-fermé forcées par le niveau de la mer et le vent : exemple de l'étang de Berre
}

\author{
Caroline PAUGAM ${ }^{1}$, Samuel MEULE ${ }^{2}$, Damien SOUS ${ }^{1,3}$, \\ Vincent FAURE ${ }^{4}$, Vincent REY ${ }^{1}$
}

1. Université de Toulon, AMU, CNRS/INSU, IRD, Mediterranean Institute of Oceanography (MIO), UM AMU 110, Toulon, France.

caroline.paugam@mio.osupytheas.fr ; rey@univ-tln.fr ; sous@univ-tln.fr

2. Aix Marseille Université, CNRS, IRD, INRA, Collège de France, CEREGE, Aix-enProvence, France. meule@cerege.fr

3. Université de Pau et des Pays de l'Adour, E2S UPPA, SIAME - MIRA, Anglet.

4. GIPREB, Berre l'étang.

faure.gipreb@gmail.com

\section{Résumé :}

En Méditerranée, le marnage est faible, et les effets barométriques, liées aux régimes dépressionnaires, contribuent de façon prépondérante aux surcotes. Dans les bassins semi-fermés, des phénomènes de basculement des plans d'eau peuvent s'ajouter aux variations du niveau, du fait de la contrainte du vent sur la surface. Dans l'étang de Berre, le manque de données continues de niveau sur le long terme ne permettait pas d'avoir une appréciation quantitative des phénomènes contribuant aux variations du niveau d'eau. Le réseau d'instrumentation HTM-NET, composé actuellement de 16 stations de mesure en Provence dont deux sur l'étang de Berre, dans les ports du Jaï et de Saint-Chamas, permet d'acquérir des données de niveau. Une station du réseau est également installée dans le port de Carro au cap Couronne en méditerranée. Nous pouvons observer d'une part une évolution du niveau d'eau dans l'étang forcée par les variations du niveau de la mer qui se transmettent par le canal de Caronte : l'effet de la marée astronomique est perceptible avec un marnage d'environ $5 \mathrm{~cm}$, et le niveau moyen suit de façon systématique les évolutions du niveau de la mer associées aux effets barométriques, inférieurs à $45 \mathrm{~cm}$. A ce forçage par la mer, s'ajoute un basculement du plan d'eau, du même ordre de grandeur, entre le nord et le sud de l'étang, par des conditions de vent fort. Des ondes de seiches dans les ports du Jaï et Saint-Chamas sont mises en évidence : une seiche d'environ 100 min est retrouvée dans les deux sites, correspondant à la résonance selon le grand axe de l'étang. D'autres seiches sont en revanche propres à des phénomènes de résonance selon le grand étang d'une part et dans l'étang de Vaïne d'autre part. Les évènements de seiches sont corrélés à la présence de vent fort et la création d'un basculement dans l'axe du bassin.

Mots-clés : Basculement, Seiches, Surcotes, Niveaux d'eau, Bassin semi-fermé. 


\section{Thème 1 - Hydrodynamique côtière}

\section{Introduction}

\subsection{Les seiches}

Les seiches sont des oscillations stationnaires à l'origine de variations du niveau d'eau. Elles peuvent être observées dans des lacs, des baies, des estuaires ou encore des ports qui sont ouverts sur la mer. D'abord plurinodales s'amortissant en ondes de mode de plus en plus faible, les ondes uninodales sont les dernières à se manifester. Ces variations d'eau dans des ports peuvent entraîner des surcotes de plusieurs centimètres et d'importants dégâts.

La longueur du bassin correspond à la moitié de la longueur d'onde de la seiche dans un bassin fermé et au quart de la longueur d'onde en bassin ouvert. Ainsi, les caractéristiques des seiches dépendent de la configuration du bassin. En revanche, leur génération peut être variée selon le site étudié : variations de pression atmosphérique (DE JONG et al., 2003, dans le port de Rotterdam et ARDHUIN et al., 2010, à Port Tudy), instabilités océaniques (CANDELA et al., 1999, dans le détroit de Sicile), ondes infra-gravitaires (OKIHIRO et al., 1993) ou encore par des tsunamis (PELINOVSKY et al., 2001, en côte d'Azur et ICHINOSE et al., 2000, dans le Lac Tahoe en Californie-Nevada).

\subsection{Basculement de plan d'eau}

En eaux peu profondes, en condition stationnaire et sans création de courants, la contribution du vent dans la variabilité du niveau de la mer peut être étudiée de manière satisfaisante par un système d'équation 1DH (équation 1), permettant d'estimer la pente de surface en fonction de la vitesse du vent $\mathrm{W}$, du coefficient de frottement $\mathrm{C}_{\mathrm{D}}$ et $\mathrm{du}$ gradient de densité air-mer.

$\frac{\partial \zeta}{\partial x}=\frac{\tau_{s}}{\rho_{w} g h}=\frac{\rho_{a} C_{D} W^{2}}{g \rho_{w} h}$

où : $\zeta$ est la surface libre, $\tau_{\mathrm{s}}$ est à tension du vent, g est l'accélération de la gravité $(9,81$ $\left.\mathrm{m} / \mathrm{s}^{2}\right), \rho_{a}$ est la densité de l'air, $\rho_{\mathrm{w}}$ est la densité de l'eau et h la profondeur de l'eau. La tension du vent correspond à la force de cisaillement que le vent exerce sur la surface de la mer. Ce dernier est défini en fonction de la vitesse du vent $\mathrm{W}$ (équation 2).

$\tau_{s}=\rho_{a} C_{D} W^{2}$

$\mathrm{C}_{\mathrm{D}}$ dépend de la rugosité de la surface marine et de la stabilité de la couche limite atmosphérique. Ce coefficient est variable mais est de l'ordre de grandeur de 1,2.10-3. Le Golfe du Lion est exposé à des vents forts : mistral et tramontane. Ainsi, les bassins semifermés provençaux sont propices à l'étude des variations du niveau d'eau induit pas le vent et par les seiches. Notre site d'étude est l'étang de Berre, une lagune située dans les Bouches-du-Rhône de profondeur moyenne de 6 mètres et maximale de 10 mètres. Il est constitué de deux parties: le grand étang à l'ouest et l'étang de Vaïne à l'est, séparés par un haut fond. Le grand axe du bassin (nord-ouest/sud-est) mesure environ $20 \mathrm{~km}$. L'étang est relié à la mer Méditerranée par le canal de Caronte situé au sud-ouest du bassin. Le 


\section{XVIèmes Journées Nationales Génie Côtier - Génie Civil \\ Le Havre 2020}

pourtour de l'étang, urbanisé et industrialisé, fait environ $75 \mathrm{~km}$ et compte dix ports dédiés à la pêche et à la plaisance. Il n'existe pas pour le moment d'études permettant de connaître les évènements responsables de la génération de seiches dans l'étang de Berre, ni le lien entre ces dernières et les basculements du plan d'eau provoqués par la tension de surface lors de vents forts. Notre étude a pour but de mettre en évidence les évènements précédemment définis dans l'étang de Berre, et essayer de comprendre leur génération.

\section{Matériel et méthodes}

Cette étude s'appuie sur des données de niveaux d'eau provenant du réseau d'observation HTM-NET (https://htmnet.mio.osupytheas.fr) ainsi que sur les données météorologiques de Météo-France à Marignane, (voir figure 1).

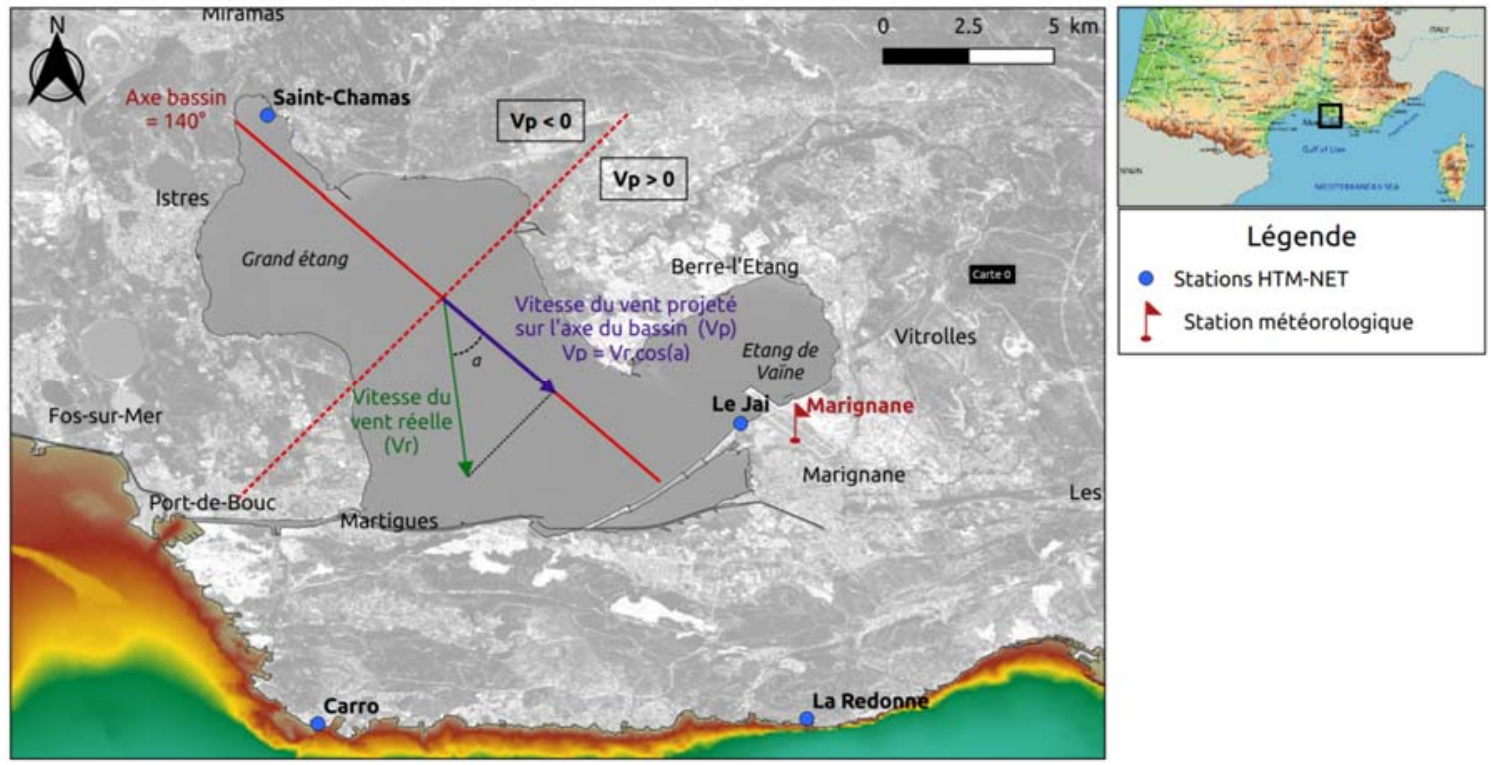

Figure 1. Stations HTM-NET et Météo-France dans l'étang de Berre et méthode de projection de la vitesse du vent dans l'axe du bassin.

\subsection{Données de niveaux d'eau}

Initialement implanté dans le Var depuis 2013, le réseau d'observation HTM-NET s'est densifié et s'est étendu dans les Bouches-du-Rhône. 16 stations fournissent des données de température et pression dans l'air et dans l'eau dans le long terme, ayant une période d'échantillonnage de $2 \mathrm{mn}$. Les niveaux d'eau sont calculés grâce à deux piézomètres, l'un immergé et l'autre émergé, permettant une correction de la pression atmosphérique sur la variation du niveau d'eau. Depuis février 2019, deux stations sont implantées dans l'étang de Berre : une dans le port de Saint-Chamas dans le grand étang, et une dans le port du Jaï en limite de l'étang de Vaïne. Les variations du niveau d'eau à l'extérieur de l'étang sont fournies par une station à Carro au cap Couronne. 


\section{Thème 1 - Hydrodynamique côtière}

\subsection{Données météorologiques}

Les données météorologiques issues des messages internationaux d'observation de surface (SYNOP) de l'Organisation Météorologique Mondiale (OMM) ont été utilisées. Le réseau permet d'obtenir les données météorologiques tri-horaires de la station située à Marignane. La rose des vents des données météorologiques de Marignane de février 2019 à février 2020 met en évidence les deux types de vent dominants : nord-ouest (mistral) et sud-est (figure 2). Ce jeu de données est représentatif des conditions générales du vent à Marignane.
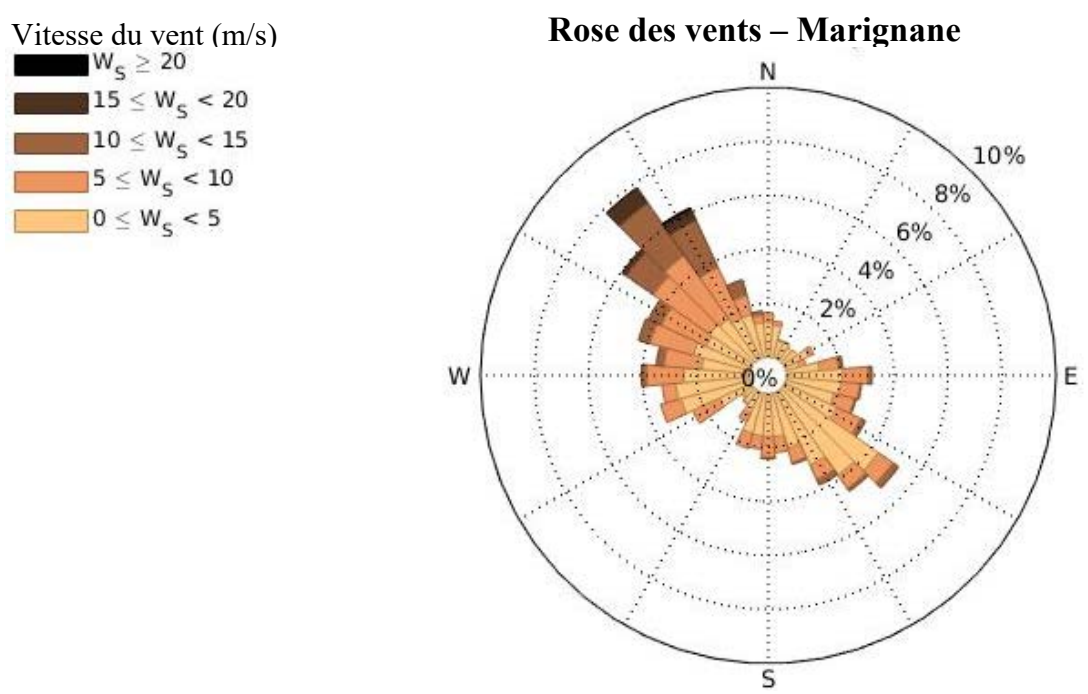

Figure 2. Rose des vents à Marignane de février 2019 à février 2020.

\subsection{Caractérisation du basculement de la surface libre}

Les données de niveaux d'eau échantillonnées toutes les $2 \mathrm{mn}$ sont moyennées par tranches de 3 heures. Ainsi, un niveau moyen toutes les 3 heures aux deux stations est utilisé pour cette étude. On définit le basculement comme la différence de niveaux d'eau entre le port du Jaï et le port de Saint-Chamas. Pour une pente de la surface libre positive du nord-ouest vers le sud-est, le basculement sera positif, et inversement. Le basculement est ensuite représenté en fonction de la composante de la vitesse du vent dans l'axe de l'étang de Berre $\left(140^{\circ}\right)$. Un évènement de mistral provoquera une vitesse de vent positive et un vent de sud-est une vitesse négative (figure 1).

\section{4 Étude des seiches}

Dans un premier temps, une analyse de Fourier de l'ensemble du jeu de données a permis de déterminer des pics d'énergie correspondants à des événements de seiches. Les fréquences associées ainsi que les fréquences minimales et maximales délimitant les pics ont été déterminés. 


\section{XVIèmes Journées Nationales Génie Côtier - Génie Civil \\ Le Havre 2020}

Les seiches ont ensuite été étudiées par intégration des pics d'énergie entre les fréquences minimale et maximale des pics, sur une fenêtre glissante de 12 heures toutes les 3 heures. Ainsi, il est possible d'observer l'évolution temporelle des seiches en fonction des conditions de vent.

\section{Résultats}

\subsection{Variation du niveau dans l'étang de Berre}

Le niveau d'eau dans l'étang de Berre est forcé par la marée via le canal de Caronte (figure 3). Le marnage est d'environ $20 \mathrm{~cm}$ au port de Carro (figure 1) et est de $5 \mathrm{~cm}$ à l'intérieur de l'étang. On observe un déphasage de la marée d'environ 140 minutes entre la mer et l'étang. Les variations de pression atmosphérique provoquent des variations de niveau d'eau en mer, par effet de baromètre inverse. Dans l'étang de Berre, les surcotes et décotes atmosphériques observées dans le port de Carro se transmettent également dans l'étang et sans atténuation via le canal de Caronte (figure 3). Sur l'année d'enregistrement, la pression atmosphérique a varié de 990,6 à 1035,5 hPa à Marignane. Cela représente des variations de niveaux de $45 \mathrm{~cm}$ dans l'étang de Berre dans l'hypothèse isostatique.

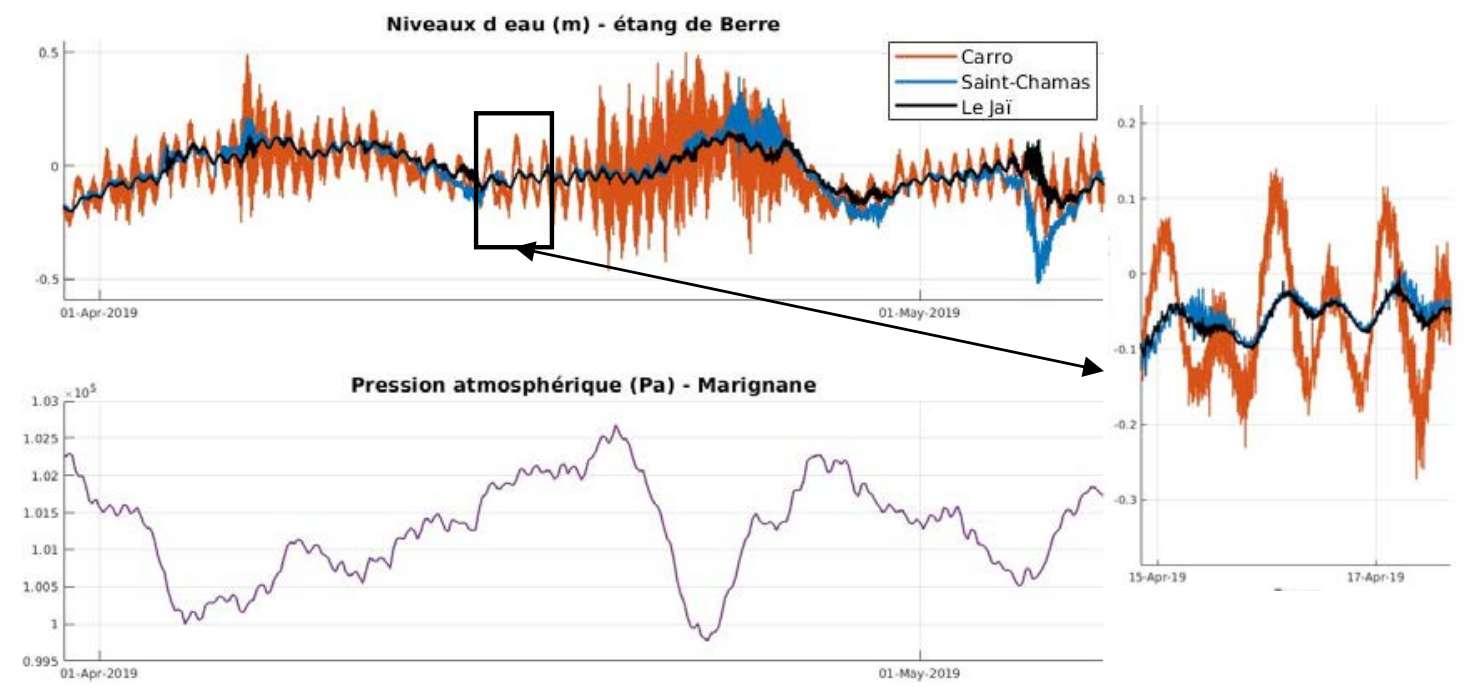

Figure 3. Niveaux d'eau aux ports de Saint-Chamas, du Jaï et de Carro.

\subsection{Basculements de la surface de l'étang de Berre}

Le basculement de la surface libre de l'étang de Berre est bien corrélé avec la présence de vent fort (figure 4) et est symétrique en cas de mistral ou de vent de sud-est. Lors d'un mistral de $17 \mathrm{~m} / \mathrm{s}$ dans l'axe de l'étang, une pente de $1 \mathrm{~cm} / \mathrm{km}$ est observée, ce qui est moins important que les résultats de LEREDDE et al. (2002) qui avaient obtenu une pente de $2 \mathrm{~cm} / \mathrm{km}$ en utilisant un modèle de circulation 3-D dans l'étang de Berre. L'équation 


\section{Thème 1 - Hydrodynamique côtière}

1 utilisée avec un $C_{D}$ constant égal à $1,2.10^{-3}$ sous-estime les valeurs de basculement et n'est donc pas un modèle représentatif pour l'étang de Berre (figure 4).

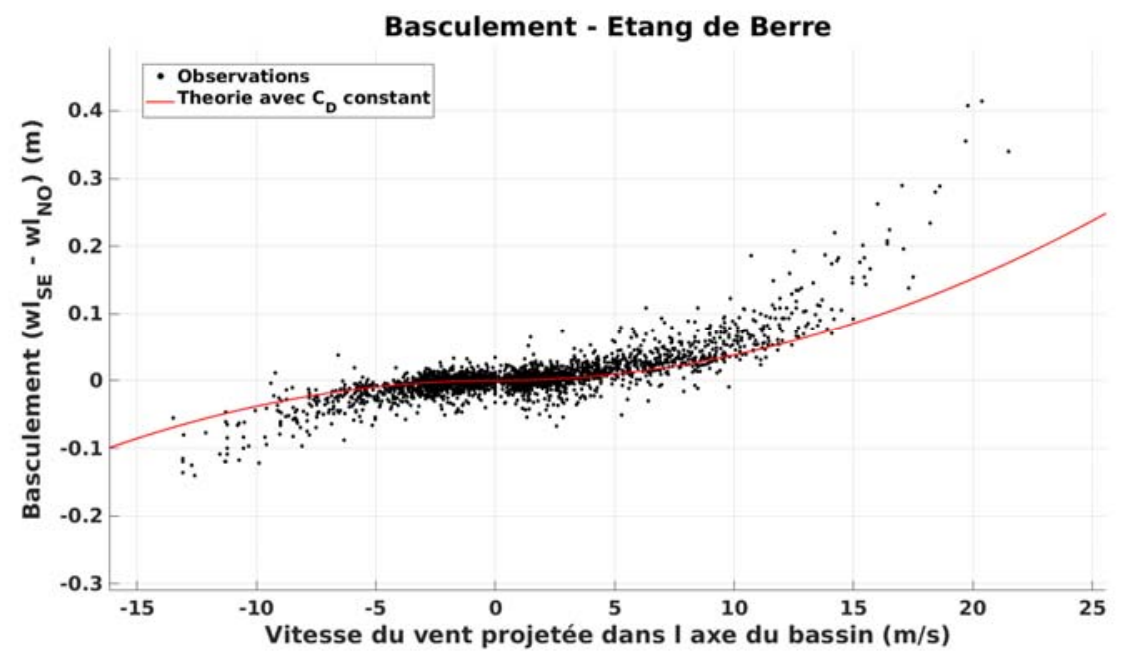

Figure 4. Basculement observé entre Le Jaï et Saint-Chamas en fonction de la vitesse $d u$ vent projetée selon l'axe de l'étang et basculement théorique avec $C_{D}=1,2 \cdot 10^{-3}$.

\subsection{Seiches dans l'étang de Berre}

Dans le port de Saint-Chamas, 8 pics d'énergie appartenant à la classe des très basses fréquences sont observés : 8.5, 13, 22.5, 27, 35, 45.5, 63.5 et $100 \mathrm{~min}$. Dans le port du Jaï, 6 seiches de fréquences 11.5, 15, 19, 22, 31 et 100 min sont observées. La seiche de $100 \mathrm{~min}$ est provoquée par une résonance dans le grand axe nord-ouest/sud-est de l'étang et est retrouvée par les deux stations. En revanche, les autres seiches ne sont pas communes aux deux sites possiblement du fait de leur séparation par un haut fond entre le grand étang et l'étang de Vaïne. La figure 5 représente l'évolution temporelle des pics d'énergie intégrés aux différentes fréquences de seiches pour les deux stations. Les évènements de seiches sont corrélés aux évènements de vent fort et aux basculements de la surface de l'eau.

\section{Discussions et perspectives}

Les données d'observation des niveaux d'eau du réseau HTM-NET de février 2019 à février 2020 nous ont permis de mettre en évidence la présence de seiches ainsi que des basculements de la surface libre par le vent dans l'étang de Berre. Le niveau d'eau dans l'étang est forcé par les variations de pression atmosphérique qui se répercutent via le canal de Caronte sans atténuation d'amplitude. De plus, la marée est également transmise avec une atténuation d'environ $75 \%$. Lors d'évènements de vents forts, la tension du vent sur la surface est responsable de la création d'une pente de la surface libre dans l'axe de la direction du vent et est responsable de la création d'un basculement. Ce dernier est 


\section{XVIèmes Journées Nationales Génie Côtier - Génie Civil \\ Le Havre 2020}

symétrique en cas de mistral ou de vent d'est/sud-est et peut être de l'ordre de $40 \mathrm{~cm}$ pour un vent de $20 \mathrm{~m} / \mathrm{s}$ dans l'axe du bassin.

L'analyse des spectres d'énergie ont mis en évidence la présence de seiches dans les ports du Jaï et Saint-Chamas. Une seiche d'environ 100 min est retrouvée dans les deux sites et semble correspondre à la résonance selon le grand axe nord-ouest/sud-est de l'étang. En revanche, d'autres seiches ne sont pas communes et sont issues de la résonance dans le grand étang pour les seiches de Saint-Chamas et l'étang de Vaïne pour le Jaï. Les évènements de seiches sont corrélés à la présence de vent et la création d'un basculement dans l'axe du bassin. Nous pouvons supposer que lors d'un évènement de vent fort, un basculement se crée et lorsque le plan d'eau retourne à l'équilibre lors de la diminution de la vitesse du vent, des ondes de résonance se créent. Les diverses configurations de résonance pourraient être expliquées en comparant ces résultats à ceux du modèle numérique TELEMAC 3D mis en place dans l'étang de Berre par le GIPREB (Groupement d'intérêt public pour la réhabilitation de l'étang de Berre). L'étude des basculements en fonction du vent permettra de mettre en place une formulation du coefficient de frottement qui pourra être utilisée dans le modèle numérique.

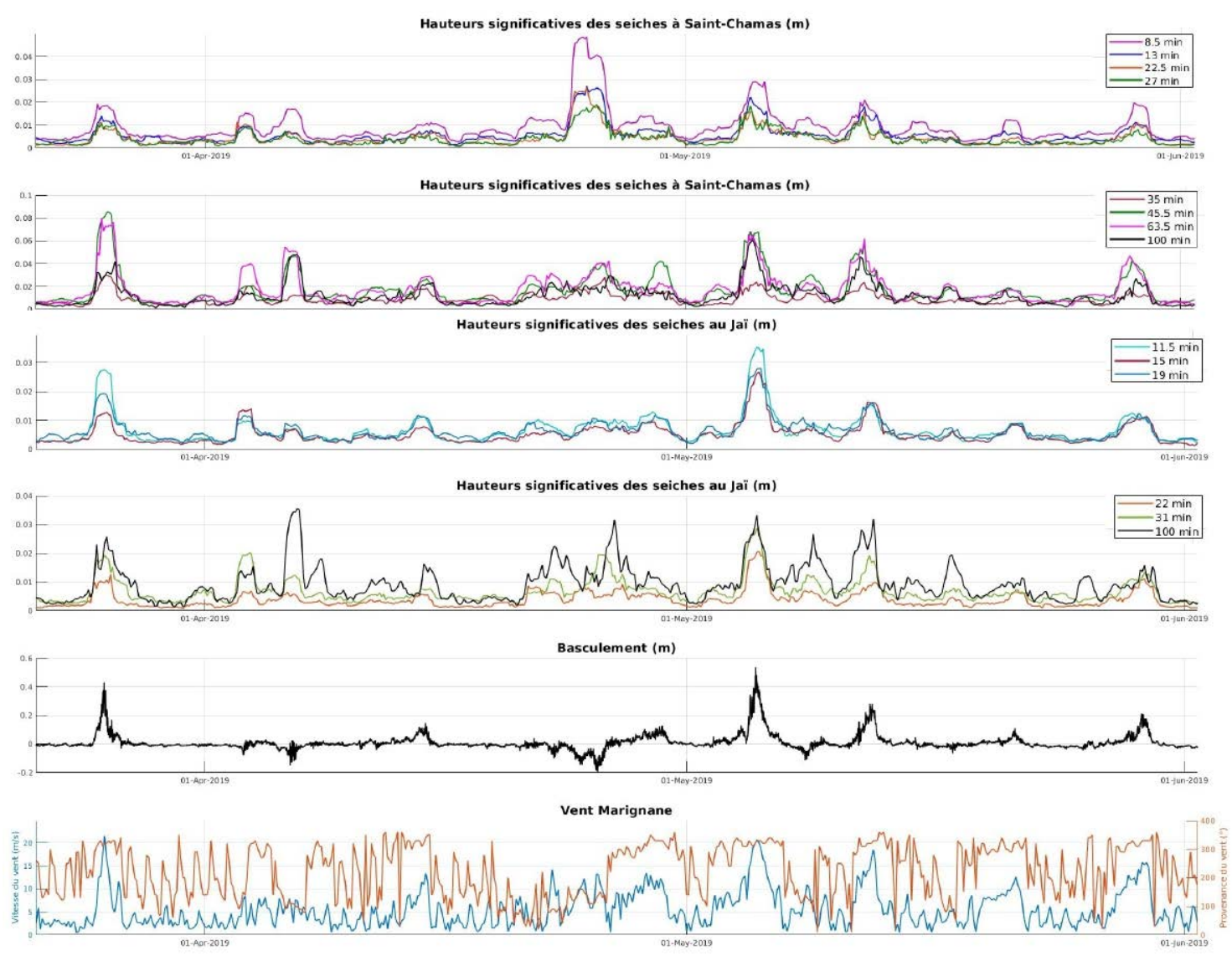

Figure 5. Hauteurs significatives des seiches aux ports de Saint-Chamas et du Jaï, basculement de la surface libre et conditions de vent. 


\section{Thème 1 - Hydrodynamique côtière}

\section{Remerciements}

Le programme d'observation HTM-NET (https://htmnet.mio.osupytheas.fr) a bénéficié de co-financements récurrents depuis 2013 de la part du CNRS/INSU, dans le cadre du programme national « Systèmes d'Observations et d'Expérimentation pour la Recherche en Environnement » sur le long terme (SOERE), Littoral -Trait de Côte, puis de ILICODYNALIT et de la communauté d'agglomération Toulon Provence Méditerranée (TPM). Le Conseil Départemental des Bouches-du-Rhône (CD13) est remercié pour les autorisations d'implantation des stations dans son département, le SHOM pour sa collaboration et Météo France pour les données météorologiques.

\section{Références bibliographiques}

ARDHUIN F., DEVAUX E., PINEAU-GUILLOU L. (2010). Observation et prévision des seiches sur la côte atlantique française. XIème Journées Nationales Génie Côtier Génie Civil, Les Sables d'Olonne, 22-25 juin 2010, pp 1-8. https://doi.org/10.5150/jngcgc.2010.001-A

CANDELA J., MAZZOLA S., SAMMARI C., LIMEBURNER R., LOZANO C.J., PATTI B., BONANNO A. (1999). The "Mad Sea" Phenomenon in the Strait of Sicily. Journal of Physical Oceanography, Vol. 29, pp 2210-2231. https://doi.org/10.1175/15200485(1999)029<2210:TMSPIT >2.0.CO;2

DE JONG M.P.C., HOLTHUIJSEN L.H., BATTJES J.A. (2003). Generation of seiches by cold fronts over the southern North Sea. Journal of Geophysical Research: Oceans, Vol. 108(C4), pp 1-10. https://doi.org/10.1029/2002JC001422

ICHINOSE G.A., ANDERSON J.G., SATAKE K., SCHWEICKERT R.A., LAHREN M.M. (2000). The potential hazard from tsunami and Seiche waves generated by large earthquakes within Lake Tahoe, California-Nevada. Geophysical Research Letters, Vol. 27(8), pp 1203-1206. https://doi.org/10.1029/1999GL011119

LEREDDE Y., DEKEYSER I., DEVENON J.-L. (2002). T-S Data Assimilation to Optimise Turbulent Viscosity: An Application to the Berre Lagoon Hydrodynamics. Journal of Coastal Research, vol. 18 (3), 555-567. https://www.jstor.org/stable/4299102 OKIHIRO M., GUZA R.T., SEYMOUR R.J. (1993). Excitation of seiche observed in a small harbor. Journal of Geophysical Research: Oceans, Vol. 98(C10), pp 18201-18211. https://doi.org/10.1029/93JC01760

PELINOVSKY E., KHARIF C., RIABOV I., FRANCIUS M. (2001). Study of tsunami propagation in the Ligurian Sea. Natural Hazards and Earth System Science, Vol. 1(4), pp 195-201. https://doi.org/10.5194/nhess-1-195-2001 